\title{
The creative application of computer technology in chinese traditional music culture
}

\author{
Lan-Lan Lyu \\ School of Digital Arts and Communication, Xiamen University of Technology, Xiamen, \\ Fujian, China \\ E-mail:kkuffuff@163.com \\ www.xmut.edu.cn
}

\begin{abstract}
Emotion is the soul of music. The main goal of music is to convey human affection. Using computer to analyze music emotion is very important. Furthermore, it is helpful in music understanding, music retrieval and some other interesting music related application. Guqin, as the symbol of Chinese music, has great research value. In this paper, for the character of Chinese traditional culture, we analyzed mainly ancient Guqin music, extracting six key emotion characteristics, structuring emotion classification model based on Beyes. Experiments indicate that there were satisfactory classification results.

Keywords: Computer; Music; Guqin; Characteristics Extraction; Beyes.
\end{abstract}

\section{Introduction}

Music spread actually is the transmission of emotion. The development of Computer technology and artificial intelligence opened up a new road for music. In the modern information age, traditional music has to face a new challenge. Nowadays, Using advanced artificial intelligence technology to analyses, process and compose music has become to the hot study point. More and more experts in related field pay attention to it. E.P. Nichols programed a "Musicat" system, which can simulate the process of listening to a simple melody in the Western tonal tradition, and displays on the screen the various internal cognitive structures that form as the melody progresses in time[1]. G.Widmer and $\mathrm{W}$ Goebl made an effort to research on the formal characterisation of differences in individual performance style[2]. S. Dixon built a computer program which is able to estimate the tempo and the times of musical beats in expressively performed music in 2001[3]. Among that, the analysis and processing of music emotion is the most difficult problem. There is a very important affect to 
compose music, understand music and so on. X.Y. Sun and Y.C. Tang used a framework named information cell mixture models to automate the task of music emotion classification [4]. In [5], a model based on Bayesian networks was proposed to automatically recognize the expressive content of MIDI piano improvisations. Our work mainly focused on the emotion processing of Guqin music.

Guqin is the oldest Chinese stringed instrument, a symbol of Chinese music. It was the preferred musical instrument . of the renowned philosopher Confucius [6,7]. In Chinese traditional culture, a well-educated scholar was expected to master four arts including Guqin. In classical age, Guqin was the most revered Chinese musical instrument and a symbol of high culture. However, only about 3000 Guqin music handed down, and fewer than two thousand people can play it nowadays. Our research is the first of its kind to apply artificial intelligence to process emotion of Guqin music.

\section{Emotional Characteristics Extraction}

Unlike western notation (only records pitch and duration)[8], Chinese Guqin music adopts tablature, which mainly records fingering, different fingerings can generate different timbres, and the rich timbre of Guqin mainly depends on the protean fingerings of left and right hands. Therefore, it is feasible to study Guqin music from the view of tablature. Under the Chinese traditional cultural background, we divide the emotion of Guqin music into eight basic categories and extract six key emotion features: tonality, major interval, rhythm, glissando, harmonic and timbre, from them. We use $f_{i}(i=1,2, \ldots, 6)$ to record them respectively.

\subsection{Tonality}

We give different impact factors for different tonalities according to their emotions. Given $m_{i}$ is certain tonality, then, the inverse is taken as the impact factor $f_{1}$. Eq.(1).

$$
f_{1}=\frac{1}{m_{i}}, \quad m_{i}=\mathrm{i}, \quad(\mathrm{i}=1, \ldots, 6)
$$

\subsection{Timbre}

Different note has different timbre, which can be determined by chord, finger and play method. First, different chord was given to a different value. Then, by played using finger or fingernail, we sure a value 1 or -1 , which can express emotion in a certain extent. At last, the frequency of three basic tones in the 
whole music was considered to obtain a feature value of the whole music. $g_{1}$ (Zhifa) means play method of right hand, which is 1 or $-1,1$ means bright emotion, -1 is opposite. $\boldsymbol{x}_{\boldsymbol{j}}$ means the jst chord. Furthermore, we can obtain the second characteristic $f_{2}$ by Eq. (2).

$$
\begin{aligned}
& \mathrm{f}_{2}=\mathrm{g}_{1}(\text { zhifa }) \sum_{\mathrm{i}=1}^{\mathrm{n}} \mathrm{fan}_{\mathrm{i}} /\left[\mathrm{x}_{\mathrm{j}} \sum_{\mathrm{j}=1}^{\mathrm{n}}\left(\mathrm{san}_{\mathrm{i}}+\mathrm{an}_{\mathrm{i}}\right)\right] \\
& g_{1}(z h i f a)= \begin{cases}1 & \text { zhifa } \in(" 110 ", " 113 ", " 115 ", " 117 ") \\
-1 & \text { zhifa } \in(" 111 ", " 112 ", " 114 ", " 116 ")\end{cases} \\
& x_{j}=j, \quad(j=1, \ldots, 7)
\end{aligned}
$$

\subsection{Major interval}

Melodic interval plays an important role in music. Major interval appears few in other music[9,10]. The mainly reason is that major interval is not too smooth and fluent, which make people uncomfortable. However, it is some common in Guqin. Its unique tone is very close to people's sound, and has prodigious infection power. Not only that, Major interval also can make emotion more rich. We use $n$ to record the total number of notes. $g_{2}\left(\right.$ pitch $_{i}$, pitch $\left._{i+1}\right)$ means pitch interval between two neighbor notes. If it equal or greater than octave, the value will be given 1, or else, 0 . Therefore, $f_{3}$ can be described in Eq. (3).

$$
\begin{array}{r}
f_{3}=\frac{1}{n-1} \sum_{i=1}^{n-1} g_{2}\left(p_{i t c h}, \quad \operatorname{pitch}_{\mathrm{i}+\_1}\right) \\
\mathrm{g}_{2}\left(\operatorname{pitch}_{\mathrm{i}}, \quad \operatorname{pitch}_{\mathrm{i}{ }_{1}}\right)=\left\{\begin{array}{ll}
1, \mid \operatorname{pitch}_{\mathrm{i}}, & \text { pitch } \\
0, & \text { else } 1
\end{array} \mid \geq 12\right.
\end{array}
$$

\subsection{Harmonic}

Harmonic played by right hand also involves some rhythm information. For example, means Tiao five and six string successively, it is faster than Tiao five string, then Tiao six string respectively. We use $g_{3}\left(\right.$ note $\left._{i}\right)$ to statistic the total number of harmonic notes. Eq.(4) can record the value of $f_{4}$.

$$
\begin{aligned}
& \left.\mathrm{f}_{4}=\frac{1}{\mathrm{n}} \sum_{\mathrm{i}=1}^{\mathrm{n}} \mathrm{g}_{3} \text { (note }_{\mathrm{i}}\right) \\
& g_{3}\left(\text { note }_{i}\right)=\left\{\begin{array}{lc}
1, & " 120 " \leq \text { note }_{i} \leq " 130 " \\
0, & \text { else }
\end{array}\right.
\end{aligned}
$$




\subsection{Glissando}

Not only glissando can make music more rich and expressive, but also make a great contribution to rhythm. If harmonic can fast the rhythm, then glissando can slow it. For example, Yin, Nao can make pitch change, and delay rhythm in a certain extent. Therefore, this characteristic can be obtained by follow statistic. Among that, given $g_{4}\left(\right.$ note $\left._{i}\right)$ as the total number of glissando. Eq.(5) gives the computer method of Glissando.

$$
\begin{gathered}
f_{5}=\frac{1}{n} \sum_{i=1}^{n} g_{4}\left(\text { note }_{i}\right) \\
g_{4}\left(\text { note }_{i}\right)=\left\{\begin{array}{lc}
1, & 154 " \leq \text { note }_{i} \leq " 163 " \\
0, & \text { else }
\end{array}\right.
\end{gathered}
$$

\subsection{Rhythm}

Although, the kind of pure rhythm notes is few in Guqin score. The pervasive using also can affect melodic affection. Therefore, basis on the class of theoretical analysis, we divide rhythm into two classes: light slow and heavy fast. Furthermore, we give them the different value by statistic in respectively. Eq.(6). $g_{5}\left(\right.$ note $\left._{i}\right)$ means rhythm fast or slow, fast is 1 , slow is -1 , or else is 0 .

$$
\begin{aligned}
& \mathrm{f}_{6}=\frac{1}{\mathrm{n}} \sum_{\mathrm{i}=1}^{\mathrm{n}} \mathrm{g}_{5}\left(\text { note }_{\mathrm{i}}\right) \\
& g_{5}\left(\text { note }_{\mathrm{i}}\right)=\left\{\begin{array}{r}
1 \text { note }_{\mathrm{i}} \in(\text { ("056", "251", "252", "307", "311", "313" } \\
-1 \text { note } \\
0
\end{array}\right.
\end{aligned}
$$

\section{Beyes Classifer Model}

Beyes classifier model mainly bases on the theory of probability statistics, which is simple, fast and has high classification accuracy. Moreover, it is as efficient as decision tree and neural network in some cases. Therefore, in our work, according to the distribution characteristic of emotional features, we divide them into three different areas firstly, then the problem can be described as follows: Let $X=\left\{x_{1}, x_{2}, \ldots, x_{6}\right\}$ denote that sample $X$ is described by six feature vectors. The various classes of musical emotion are respectively represented by $C_{1}$, $C_{2}, \ldots, C_{8}$. Given a sample $X$, which has not been classified, if $X$ is classified into $C_{\mathrm{i}}$, there must be a value $i$ can meet $P\left(C_{\mathrm{i}} \mid X\right)>P\left(C_{\mathrm{k}} \mid X\right), 1 \leq k \leq 8$ and $i \neq k$. Assume the following definition: TotalNum means the total number of samples; ClassNum ${ }_{\mathrm{i}}$ represents the sample number of class i, let AttriClassNumij be the number of $j$-th attribute value in class $i$. 


$$
\begin{aligned}
& \mathrm{P}\left(\mathrm{C}_{\mathrm{i}}\right)=\frac{\text { ClassNum }_{\mathrm{i}}}{\text { TotalNum }} \\
& \mathrm{P}\left(\mathrm{x}_{\mathrm{j}} \mid \mathrm{m}_{\mathrm{i}}\right)=\frac{\text { AttriclassNum }_{\mathrm{ij}}}{\text { ClassNum }_{\mathrm{i}}} \\
& \mathrm{P}\left(\mathrm{C}_{\mathrm{i}} \mid \mathrm{x}_{\mathrm{j}}\right)=\frac{\mathrm{P}\left(\mathrm{C}_{\mathrm{i}}\right) \mathrm{P}\left(\mathrm{x}_{\mathrm{j}} \mid \mathrm{C}_{\mathrm{i}}\right)}{\sum_{\mathrm{i}=1}^{8} \mathrm{P}\left(\mathrm{C}_{\mathrm{i}}\right) \mathrm{P}\left(\mathrm{x}_{\mathrm{j}} \mid \mathrm{C}_{\mathrm{i}}\right)}
\end{aligned}
$$

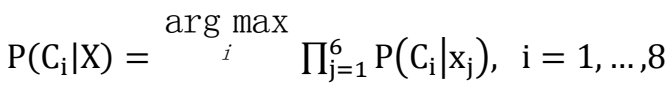

Basing on the training sets of Guqin data, we can obtain the priori probability by Eq. (7) and (8). Then, the posterior probability of attribute $j$ in class $i \mathrm{can}$ be obtained according to Beyes classification Eq. (9). Finally, the unknown class of sample X can be classified by Eq. (10). By this method, the experimental results

\begin{tabular}{|c|c|c|c|c|c|c|c|}
\hline Tonality & Major interval & Rhythm & Glissando & Harmonic & timbre & $\begin{array}{l}\text { Classification } \\
\text { result }\end{array}$ & $\begin{array}{l}\text { Correct } \\
\text { class }\end{array}$ \\
\hline 5 & 0.319767 & 0 & 0.0977011 & 0.0114943 & 0.114943 & 1 & 1 \\
\hline 4 & 0.158192 & 0.0062435 & 0.332986 & 0.0322581 & 0.0775234 & 1 & 1 \\
\hline 4 & 0.182674 & 0 & 0.184158 & 0.0679868 & 0.0620462 & 1 & 1 \\
\hline 7 & 0.345277 & 0.0030581 & 0.16208 & 0.155963 & -0.00611621 & 2 & 2 \\
\hline 6 & 0.142298 & -0.0117493 & 0.219321 & 0.0221932 & 0.0770235 & 2 & 2 \\
\hline 1 & 0.131902 & -0.00857143 & 0.454286 & 0.00857143 & -0.0114286 & 7 & 7 \\
\hline 3 & 0.154018 & 0.0108959 & 0.360775 & 0.104116 & 0.094431 & 7 & 7 \\
\hline 5 & 0.324324 & 0 & 0.0797872 & 0.0106383 & 0.117021 & 1 & 1 \\
\hline 9 & 0.277286 & -0.000761615 & 0.184311 & 0.0662605 & 0.0624524 & 1 & 1 \\
\hline 2 & 0.355814 & 0.00902935 & 0.0744921 & 0.0158014 & 0.0586907 & 1 & 1 \\
\hline 0 & 0.272853 & -0.00133511 & 0.226969 & 0.0307076 & 0.154873 & 5 & 5 \\
\hline 0 & 0.283019 & -0.0154083 & 0.180277 & 0.00616333 & 0.11094 & 7 & 2 \\
\hline 8 & 0.1 & 0.0149864 & 0.320163 & 0.0367847 & 0.0831063 & 6 & 6 \\
\hline 0 & 0.125475 & 0 & 0.0442804 & 0.00369004 & -0.0369004 & 7 & 7 \\
\hline 0 & 0.158184 & -0.00092509 & 0.291397 & 0.00832562 & 0.0656799 & 6 & 7 \\
\hline 0 & 0.173278 & -0.00631313 & 0.265152 & 0.0707071 & 0.0631313 & 6 & 6 \\
\hline 1 & 0.131902 & -0.0085713 & 0.454286 & 0.00857143 & -0.0114286 & 7 & 2 \\
\hline 3 & 0.154018 & 0.0108959 & 0.360775 & 0.104116 & 0.094431 & 7 & 7 \\
\hline 0 & 0.20578 & 0 & 0.107038 & 0.0172287 & 0.0377566 & 6 & 6 \\
\hline 0 & 0.214286 & -0.00746269 & 0.365672 & 0.00746269 & -0.0223881 & 3 & 3 \\
\hline 0 & 0.232484 & -0.00593472 & 0.424332 & 0.00890208 & -0.0178042 & 1 & 1 \\
\hline 0 & 0.213542 & 0 & 0.329016 & 0.0259067 & 0.0544041 & 3 & 3 \\
\hline 0 & 0.25974 & 0.0149254 & 0.460554 & 0.0234542 & 0.0618337 & 3 & 3 \\
\hline 0 & 0.177632 & -0.00108932 & 0.22549 & 0.00980392 & 0.083878 & 6 & 7 \\
\hline 0 & 0.183932 & -0.0105882 & 0.221176 & 0.0611765 & 0.0541176 & 6 & 6 \\
\hline 0 & 0.204545 & 0.00724638 & 0.263285 & 0.0193237 & 0.0628019 & 3 & 3 \\
\hline 0 & 0.20354 & 0.00923361 & 0.11265 & 0.0369344 & -0.0304709 & 7 & 7 \\
\hline 0 & 0.204023 & 0.0286561 & 0.212451 & 0.0266798 & 0.027668 & 2 & 2 \\
\hline 0 & 0.209125 & -0.00764331 & 0.178344 & 0.011465 & 0.0980892 & 6 & 6 \\
\hline 4 & 0.0574713 & -0.00326493 & 0.33209 & 0.0303172 & 0.0750933 & 1 & 1 \\
\hline 4 & 0.148148 & 0.000928505 & 0.145775 & 0.0696379 & 0.0557103 & 1 & 1 \\
\hline 0 & 0.213592 & 0.011846 & 0.269497 & 0.0088845 & 0.088845 & 3 & 3 \\
\hline 1 & 0.0623229 & -0.0166667 & 0.0857143 & 0.278571 & 0.0809524 & 7 & 7 \\
\hline 1 & 0.0983607 & -0.0133333 & 0.0906667 & 0.336 & 0.0853333 & 7 & 7 \\
\hline 1 & 0.118457 & -0.027972 & 0.121212 & 0.310023 & 0.0722611 & 7 & 7 \\
\hline 0 & 0.0812808 & 0.0111111 & 0.306667 & 0.0177778 & 0.0577778 & 4 & 3 \\
\hline 0 & 0.089613 & 0.012987 & 0.368421 & 0.038961 & 0.0362269 & 4 & 4 \\
\hline 0 & 0.116379 & -0.00910273 & 0.261378 & 0.0715215 & 0.0624187 & 6 & 6 \\
\hline 0 & 0.118812 & -0.00675676 & 0.398649 & 0.00675676 & 0.0675676 & 4 & 4 \\
\hline 0 & 0.125326 & 0.00145773 & 0.385568 & 0.0459184 & 0.0750729 & 4 & 4 \\
\hline 0 & 0.129562 & 0.0223752 & 0.282272 & 0.060241 & 0.129088 & 5 & 6 \\
\hline
\end{tabular}
are showed in Table 1, we can see that the accuracy rate obtains $85.36 \%$.

Tab. 1 Experiment results of Beyes Classifier 


\section{Conclusion}

Chinese Guqin music adopts tablature, which records where and how to play every note. Hence, tablature provides an effective carrier for researching Guqin musical emotion. We extracted six key characteristics, and analysed their significance, then, we construct an emotion classification model using beyes. Although it is satisfied, we still need further to improve the classify accuracy. Consider the ambiguity and uncertainty of musical emotion. we are going to find a new way to dig more detailed information for emotion recognition.

\section{Acknowledgments}

This work is supported by the foundation of Guqin Music Composition by Intelligent Technology(YKJ13040R), and the Study and Practice of Creative Talent Cultivation in Music technology(JGY201549).

\section{References}

1. E.P. Nichols. Musicat: a Computer Model of Musical Listening and Analogy-making. Submitted for the degree Doctor of Philosophy. Indiana University. (2012).

2. G.Widmer, W Goebl. Computational Models of Expressive Music Performance:The State of the Art, Journal of New Music Research, Routledge, London, (2004),pp.203-216.

3. S. Dixon, Automatic extraction of tempo and beat from expressive performances, Journal of New Music Research, Routledge, London, (2001).pp. 39-58.

4. X.Y. Sun, Y. C. Tang, Automatic Music Emotion Classification Using a New Classification Algorithm. 2009 Second International Symposium on Computational Intelligence and Design.Changsha,( 2009).pp. 540-542.

5. Mion, Application of Bayesian networks to automatic recognition of expressive content of piano improvisations. Proc. Stockholm Music Acoustics Conf. (2003), pp.557-560.

6. C.H.Liu, Guqin Yin Shu Lun. Jiangsu Literature Press, Jiangsu,(2002)

7. M.M. Ye, Guqin music Art. The Commercial Press, Beijing, (1992).

8. Z.W.Liu, The aesthetic discussion of Chinese and Western music structure. Music Research., People's Music Publishing House.,Beijing. (2003), pp.316.

9. Y.b.,Li.,The performance role of melodic interval. Art Education. Zhongguo Wen Hua Bao She, Beijing.,pp. 66-68. 
10. Y.X. Du, Zhongguo Chuan Tong Yue Li Jiao Cheng. Shanghai Conservatory Of Music press, Shanghai,(2004). 